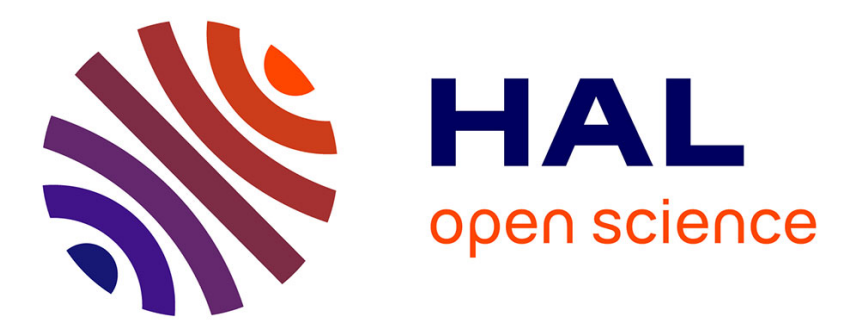

\title{
Construction et évaluation de l'effet établissement : le travail des collèges
}

\author{
Olivier Cousin
}

\section{To cite this version:}

Olivier Cousin. Construction et évaluation de l'effet établissement: le travail des collèges. Revue Française de Pédagogie, 1996, 115 (1), pp.59-75. 10.3406/rfp.1996.1201 . hal-02091736

\section{HAL Id: hal-02091736 https://hal.science/hal-02091736}

Submitted on 12 Apr 2019

HAL is a multi-disciplinary open access archive for the deposit and dissemination of scientific research documents, whether they are published or not. The documents may come from teaching and research institutions in France or abroad, or from public or private research centers.
L'archive ouverte pluridisciplinaire HAL, est destinée au dépôt et à la diffusion de documents scientifiques de niveau recherche, publiés ou non, émanant des établissements d'enseignement et de recherche français ou étrangers, des laboratoires publics ou privés. 


\title{
Construction et évaluation de l'effet établissement : le travail des
} collèges

\author{
Mr Olivier Cousin
}

\section{Résumé}

A survey was conducted among the pupils of twelve junior high schools from the second to the fourth year. Selection and orientation mecanisms were analysed in each phase of the school system, with the help of a multiva- riated regression method. Though some variable have a strong predictive capacity, concerning school success (social origin, age, sex), pupils selection differs according to the schools. So colleges produce (partly) school failure. Though there is a strong correlation between the social composition of a school and pupils orientation, some results are significantly different. This is due to a school effect : the selectivity of a school is not only the result of its social composition, it is also the product of the actions and behaviours of the actors (leaders, teaching staff).

Social cohesion differs according to the schools, the least selective are those which have collective goals and do not separate educationals tasks and pedagogical acts.

\section{Citer ce document / Cite this document :}

Cousin Olivier. Construction et évaluation de l'effet établissement : le travail des collèges. In: Revue française de pédagogie, volume 115, 1996. Les collèges. pp. 59-75;

doi : 10.3406/rfp.1996.1201

http://www.persee.fr/doc/rfp_0556-7807_1996_num_115_1_1201

Document généré le 07/06/2016 


\section{Construction et évaluation de l'effet établissement : le travail des collèges*}

Olivier Cousin

Les élèves de douze collèges ont été suivis dans leur scolarité de la $5^{\theta}$ à la fin de la $3^{e}$. Les mécanismes de la sélection et de l'orientation ont été analysés à chaque palier du système scolaire à partir d'une étude statistique complexe utilisant la méthode de l'analyse de régression multiple. L'étude de la sélection montre plusieurs phénomènes : bien qu'il existe des variables particulièrement discriminantes ayant une forte valeur prédictive de la réussite scolaire (origine sociale, âge, sexe), la sélection des élèves varie selon les établissements. Les collèges produisent donc, en partie, l'échec scolaire. Bien qu'il existe une corrélation importante entre la composition sociale de l'établissement et l'orientation des élèves, on constate des écarts à la moyenne importants. Ainsi il existe un effet établissement, ce qui laisse à penser que la sélectivité d'un établissement ne se réduit pas à sa composition sociale, elle est aussi le produit des actions et des conduites des acteurs (équipe de direction, personnel éducatif et professeurs). La nature de la cohésion sociale varie selon les collèges et ce sont les établissements qui réussissent à définir collectivement leurs objectifs et à dépasser la division entre tâches éducatives et actions pédagogiques qui se révèlent les moins sélectifs.

B ien que l'école reste une institution centralisée et chargée de donner le même savoir à tous les élèves, elle supporte dans son organisation même une part de différenciation entre les établissements. Officiellement depuis 1989, et avant pour un grand nombre d'entre eux, les établissements scolaires doivent, à partir d'un diagnostic de leur situation, établir un projet afin de remédier aux problèmes rencontrés. Ils possèdent donc une part d'autonomie. Chaque établissement élabore une politique afin de répondre à la situation à laquelle il est confronté. Les collèges ont été les premiers à bénéficier de cette marge de manœuvre et à se lancer dans ce que l'on nomme les "politiques d'établissements".

Introduire l'établissement, comme variable explicative, dans la problématique de l'inégalité des chances signifie qu'il ne s'agit plus seulement d'évaluer les chances de réussite des jeunes glo. balement mais de se demander si ces chances sont les mêmes quel que soit le collège. Ainsi, la 
problématique de l'échec scolaire adopte le principe de l'analyse écologique, jusqu'ici essentiellement appliquée à la sociologie électorale. Les mécanismes de production du parcours scolaire des élèves sont donc appréhendés à partir des modes d'organisation des établissements scolaires. De l'organisation des établissements, nous retiendrons principalement les objectifs pédagogiques et éducatifs fixés par l'établissement à travers son projet ainsi que l'engagement et la participation des acteurs dans la politique de l'établissement. Nous faisons l'hypothèse suivante : toutes choses égales par ailleurs, l'orientation des élèves dépend de l'établissement, et la construction sociale de l'établissement rend, en partie, compte de ces variations de sélectivité. L'établissement existe comme une entité sociale à part entière en fonction de sa capacité à construire une norme collective partagée par l'ensemble des intervenants. Celle-ci s'élabore à partir de la définition que chaque acteur donne de la situation et de l'interprétation qu'il donne de son rôle dans un ensemble social. L'hypothèse d'un effet établissement (1) vise donc à répondre à une double question : à population équivalente existe$t$-il des variations dans les logiques d'orientation entre les établissements? En quoi l'organisation sociale de l'établissement permet-elle de rendre compte de ces variations (2) ?

Les collèges se distinguent à la fois par leur sélectivité et par leurs politiques (3). Si dans l'ensemble, ce sont les enfants appartenant aux couches supérieures et les établissements au recrutement social le plus favorisé qui réussissent le mieux, on constate qu'à population équivalente les collèges ne connaissent pas les mêmes résultats. La sélectivité des établissements ne se réduit donc pas à leur composition sociale. L'échec scolaire est aussi en partie produit par l'établissement. Les politiques des établissements varient significativement d'un collège à l'autre et ce sont les collèges connaissant le plus fort degré de cohésion sociale entre les acteurs et le plus fort engagement des enseignants dans la politique définie par l'établissement qui se montrent les moins sélectifs et les plus équitables. Par rapport à des collèges comparables, ces établissements conduisent un plus grand nombre d'élèves en seconde trois ans après la cinquième et ils réussissent quelquefois à réduire les inégalités sociales.

Nous avons testé l'hypothèse d'un effet établissement sur un ensemble de douze collèges, entre septembre 1990 et juin 1993. Deux moments ont rythmé cette recherche. Dans un premier temps, à partir d'un suivi de l'ensemble des élèves de $5^{\ominus}$ jusqu'en fin de $3^{8}$, la production scolaire des collèges a été interprétée par rapport à l'orientation des élèves à chaque fin d'année. L'attention a été particulièrement portée sur la capacité des établissements à amener, en 3 ans, les élèves de la $5^{\mathrm{e}}$ au lycée. La sélectivité relative des collèges est évaluée par rapport à la moyenne des douze collèges retenus. Puis, dans un deuxième temps, nous avons cherché à définir les modes d'organisation des établissements à partir d'entretiens réalisés avec les principaux acteurs du système scolaire (équipe de direction, enseignants, personnel d'encadrement éducatif).

\section{LA SÉLECTIVITÉ DES COLLÈGES}

La sélectivité des établissements peut être appréciée à chaque niveau scolaire en fonction de l'orientation des élèves et de leurs notes moyennes sur l'année (4). L'échantillon de départ comprend 1516 élèves répartis sur les 12 collèges. Nous présenterons tout d'abord l'orientation générale des élèves, puis selon les établissements en fin de $5^{\theta}$ et de $3^{e}$.

\section{La $5^{\circ}$, une classe sélective}

La classe de $5^{e}$ représente le premier palier d'orientation du système scolaire français. Jusqu'au collège, l'orientation n'entre pratiquement pas en jeu dans la scolarité, les élèves passent dans la classe supérieure ou redoublent. À partir de la $5^{\mathrm{e}}$, les familles doivent "choisir", selon différents paramètres, la filière de leurs enfants. Parmi les 1516 élèves inscrits en $5^{\mathrm{e}}$, les trois quarts $(75,5 \%)$ passent en $4^{\mathrm{e}}$ et $12,5 \%$ redoublent. De la sorte, $88 \%$ des collégiens restent dans l'enseignement général. L'essentiel des élèves qui sortent de l'enseignement général part en $4^{e}$ technologique. La proportion des élèves " sortants " diminue chaque année depuis le début des années 80 en raison de la suppression des classes de CPA et CPPN.

Les résultats obtenus dans cette étude sont conformes aux données fournies par les grandes enquêtes sociologiques (5). Dans l'ensemble les garçons sont plus pénalisés que les filles qui, à note équivalente, passent toujours plus souvent dans la classe supérieure (6). Les inégalités 
sociales sont encore plus marquées que les inégalités entre filles et garçons. À note équivalente, les enfants d'ouvriers passent moins que la moyenne et vont constituer l'essentiel des effectifs des classes de LP (7). Enfin, l'âge est la variable la plus discriminante. Les élèves qui n'ont jamais pris de retard passent beaucoup plus que tous les autres, et les chances de passer en $4^{e}$ diminuent dès que les élèves prennent du retard (8). Mais ces résultats globaux cachent de grandes différences selon les établissements.

\section{L'orientation par établissement}

La sélectivité des établissements doit être interprétée de manière relative. II est nécessaire de replacer les collèges dans leur "catégorie ", un établissement est sélectif par rapport à un autre, comparable en terme de recrutement social, et non pas dans l'absolu. Cette précision vise à tester l'hypothèse d'un effet établissement, c'est-à-dire à se demander si à recrutement équivalent, l'orientation des élèves est la même dans les établissements.

\section{Des probabilités de passage très inégales}

II n'est pas étonnant de constater que l'orientation change selon les établissements puisque les collèges retenus dans cette étude ont des profils sociologiques très différents. Si, en moyenne, $75,5 \%$ des élèves passent en $4^{e}$, cette probabilité varie entre $50 \%$ pour le collège le plus sélectif en fin de $5^{e}$ et $87,5 \%$ pour le collège le moins pénalisant. Cinq collèges font significativement plus passer en $4^{\mathrm{e}}$ alors que 3 établissements sanctionnent plus sévèrement leurs élèves.

Corrélativement, il en va de même pour le redoublement et l'orientation vers le LP. Si, en moyenne, $12,5 \%$ des élèves redoublent, dans certains collèges le taux de redoublement peut s'élever jusqu'à $25 \%$, ou ne toucher que $3 \%$ des collégiens dans d'autres. Les départs vers les $4^{e}$ technologique oscillent entre $16 \%$ et $1,5 \%$, alors qu'il est de $6,5 \%$ en moyenne. Ces variations s'expliquent à la fois par le recrutement social des établissements et par les stratégies des collèges et des familles. Ainsi, dans quelques établissements l'orientation vers le LP est l'exception et les élèves, quel que soit leur niveau scolaire, redoublent. Dans d'autres, au contraire, le redoublement est l'exception. Enfin, il existe aussi des collèges qui pratiquent massivement les deux types d'orientation. C'est le cas, par exemple, d'un établissement populaire qui fait plus redoubler que la moyenne $(17 \%)$ et oriente plus souvent ses élèves en $4^{\theta}$ technologique $(10 \%)$.

Les mêmes observations peuvent être faites à partir des principales variables présentées précédemment. Selon les établissements les filles et les garçons n'ont pas les mêmes chances de succès et, surtout, dans certains cas les écarts s'accroissent alors que dans d'autres ils diminuent et quelquefois s'inversent. Le même constat s'impose quand on compare les chances de passer des élèves selon leur origine sociale ou selon leur année de naissance. II faut surtout noter l'absence de "logique " apparente. En effet, pratiquement aucun collège ne se situe de la même manière par rapport à la moyenne sur ces trois variables, comme le montre le tableau ci-dessous.

Tableau 1. - Les différents types de sélection selon les établissements par rapport à la moyenne, en fin de $5^{\theta}$

\begin{tabular}{|c|c|c|c|}
\hline & Garçons/filles & $\begin{array}{c}\text { Élève à "l'heure "/en } \\
\text { retard }\end{array}$ & $\begin{array}{l}\text { Ouvriers/cadres } \\
\text { supérieurs }\end{array}$ \\
\hline Augmentation des écarts & $\begin{array}{l}\text { CEillets, Vaughan } \\
\text { Ellington, Ravel }\end{array}$ & $\begin{array}{l}\text { Vaughan, lonesco } \\
\text { Ellington, Reinhardt } \\
\text { Chardonne }\end{array}$ & $\begin{array}{c}\text { CEillets, Le Marais } \\
\text { Ionesco } \\
\text { Ravel }\end{array}$ \\
\hline Diminution des écarts & $\begin{array}{c}\text { Genet, Chardonne } \\
\text { Mingus }\end{array}$ & $\begin{array}{c}\text { CEillets, Marais } \\
\text { Genet, Ravel } \\
\text { Mingus }\end{array}$ & $\begin{array}{l}\text { Bacchus, Renoir } \\
\text { Vaughan, Ellington } \\
\text { Chardonne, Mingus }\end{array}$ \\
\hline Inversion de tendance & Le Marais, Reinhardt & Bacchus & Reinhardt \\
\hline $\begin{array}{l}\text { Rapports identiques } \\
\text { à la moyenne }\end{array}$ & $\begin{array}{l}\text { Bacchus, Renoir } \\
\text { Ionesco }\end{array}$ & Renoir & Genet \\
\hline
\end{tabular}

Le tableau se lit de la manière suivante : par rapport à la moyenne, le collège des cEillets aggrave les écarts de probabilité de passage entre les filles et les garçons, il diminue les écarts entre les élèves "à l'heure " et les élèves trés en retard, et il aggrave les écarts entre les enfants d'ouvriers et les enfants de cadres supérieurs. 
Seul le collège C. Mingus semble " cohérent", puisqu'il réduit les écarts sur chacune des variables retenues. II faut aussi souligner qu'il n'y a pas de lien a priori entre le recrutement social et cette répartition sur ces trois variables. Parmi les établissements qui aggravent les écarts entre filles et garçons, il y a trois collèges plutôt populaires et un établissement socialement favorisé (Ravel). Il en va de même pour la sélection sociale, si deux des collèges favorisés (Chardonne et Mingus) atténuent les écarts entre les enfants d'ouvriers et les enfants de cadres, c'est aussi le cas pour des collèges populaires. Enfin, parmi les établissements qui inversent les tendances observées, c'est-à-dire qui font plus passer les garçons que les filles en $4^{e}$ ou qui conduisent plus d'enfants d'ouvriers en $4^{e}$ que d'enfants de cadres supérieurs, il n'existe pas d'unité ; des collèges populaires et des collèges favorisés sont dans ce cas de figure.

\section{Un effet établissement}

Afin d'apprécier l'effet établissement nous avons introduit une analyse de régression permettant de tester la relation entre le recrutement social des établissements et la probabilité de passer en $4^{\theta}$. Ces deux variables sont-elles indépendantes? Le graphique ci-dessous offre la possibilité de comprendre la complexité du phénomène.

Les établissements sont répartis selon la proportion d'enfants de cadres supérieurs (axes des abscisses), allant du moins favorisé au plus

\section{Graphique 1. - Taux de passage en $4^{\circ}$ par collège en fonction du nombre d'enfants de cadres supérieurs}

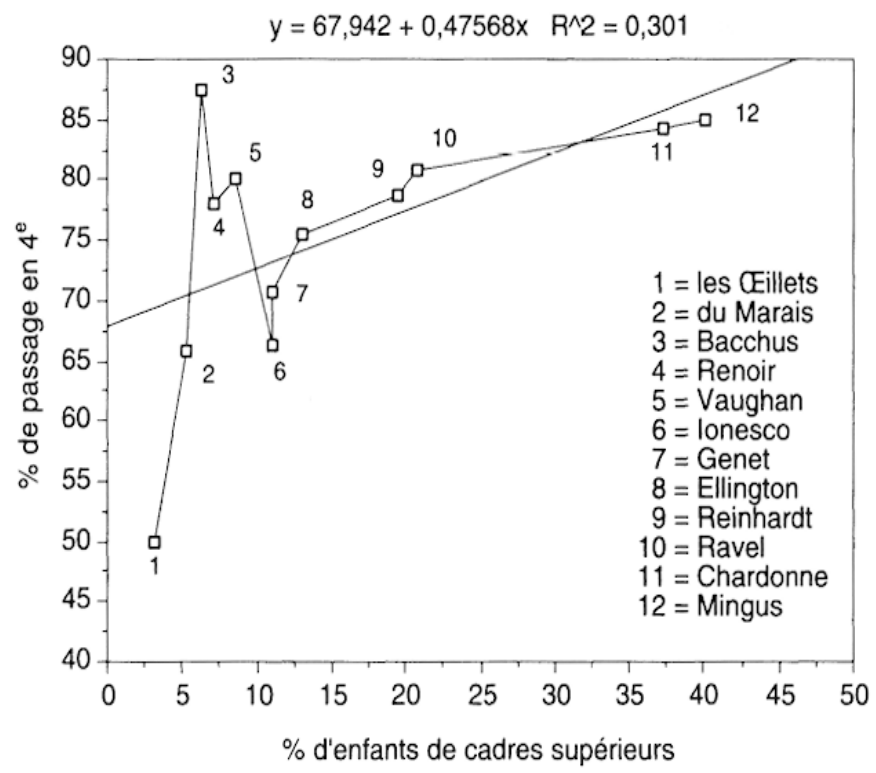

"bourgeois". $\dot{A}$ chaque établissement correspond un taux de passage en $4^{e}$ (axe des ordonnées). La visualisation de la courbe et le calcul de la droite de régression montrent qu'il existe une forte relation entre le recrutement social des établissements et la probabilité de passer en $4^{e}$ $(R=0,55 ; T=2,08)(9)$. Selon cette courbe, il n'y aurait pas a priori d'effet établissement. Or, il existe des points qui s'éloignent suffisamment de la droite de régression pour que le recrutement social des collèges ne puisse, à lui seul, rendre compte de l'orientation des élèves. Ces points représentent des cas "déviants". Ils sont ceux qui nous intéressent plus particulièrement.

On peut effectivement supposer que, parmi les établissements populaires, la sélectivité du collège 1 est "anormalement " élevée alors que celle du collège 3 est, à l'inverse, " anormalement "faible. Par ailleurs, on constate que les collèges 6 et 7 sont plus sélectifs que les collèges 4 et 5 alors qu'ils sont socialement plus favorisés. En revanche, pour les quatre établissements les plus favorisés, le lien entre la composition sociale et l'orientation reste fort. Ils respectent une certaine hiérarchie, où le collège le plus "bourgeois" des quatre est aussi le moins sélectif. Cependant, la différence entre le recrutement social et l'orientation n'est pas de la même grandeur. Le collège 12, par exemple, est socialement très favorisé (plus de $40 \%$ d'enfants de cadres supérieurs en $\left.5^{e}\right)$ alors qu'il a un taux de passage en $4^{e}$ très proche du collège 9 .

La présentation de l'orientation de fin de $5^{\mathrm{e}}$ autorise donc à rejeter l'hypothèse d'un pur effet linéaire, les catégories sociales réussissent indépendamment les unes des autres, et d'un effet de composition, plus un collège accueille une population favorisée et plus l'ensemble passe dans la classe supérieure. Ces deux hypothèses ne sont pas suffisantes pour expliquer les variations d'orientation entre les collèges. Les écarts à la moyenne observés (tableau 1 et graphique 1) laissent penser qu'on ne peut expliquer la sélection par ces seules voies. En fin de $3^{e}$, le même phénomène se reproduit mais l'ordre des établissements selon leur capacité d'amener en 3 ans les élèves de $5^{\ominus}$ en seconde est sensiblement bouleversé.

\section{La $3^{\circ}$, une classe très sélective}

Parmi les 1516 élèves qui constituaient la base de l'échantillon, 928 se retrouvent en $3^{e}$ trois ans 
plus tard $(61,5 \%)$. Près de $40 \%$ des élèves (577 individus) ont connu des problèmes entre la $5^{e}$ et la $3^{e}$. La plus grande partie d'entre eux a été soit orientée vers l'enseignement technologique, soit contrainte à redoubler. II y a aussi quelques élèves autorisés à passer dans la classe supérieure qui changent d'établissement; ce fut le cas pour 85 élèves entre la $5^{\theta}$ et la $4^{e}$. Une année plus tard, entre la $4^{e}$ et la $3^{e}$, ce sont encore 47 élèves qui quittent la cohorte sans avoir été directement pénalisés par les établissements (10).

Des deux paliers d'orientation, la classe de $3^{e}$ est la plus sélective. $1 / 3$ des élèves échouent à la fin de la $3^{\mathrm{e}}(35,5 \%)$ et se voient orientés soit en BEP $(26 \%)$, soit vers le redoublement $(9,5 \%)$. Les classes de BEP peuvent être perçues comme des classes de relégation, au même titre que les $4^{e}$ technologique, car elles sont essentiellement composées par des élèves ayant des résultats scolaires très médiocres et par les plus âgés d'entre eux ( $49 \%$ de futurs élèves de BEP obtiennent moins de 8 de moyenne générale en $3^{e}$ et $76 \%$ ont au moins une année de retard). $64 \%$ des jeunes passent en seconde. Le taux de redoublement peut paraître faible, et a fortiori celui de l'orientation en BEP très élevé. Deux raisons permettent de l'expliquer. Tout au long de la décennie 80 , le taux moyen de redoublements en fin de $3^{e}$ s'est situé autour de $13 \%$ (11) mais il tend à diminuer depuis le début des années 90 et se stabilise autour de $9 \%$ (12). Si le taux de redoublement diminue, c'est à la fois parce que les élèves accèdent de plus en plus à la classe de seconde (13) dans le cadre de la politique des " $80 \%$ d'une classe d'âge au niveau du baccalauréat " et parce que le nombre de filières a considérablement augmenté ces dernières années, particulièrement dans l'enseignement technique et professionnel. Par ailleurs, les données que nous possédons ne sont pas définitives. En effet, pour quelques élèves, l'orientation en fin de $3^{e}$ sera différente de celle que nous avons enregistrée. L'orientation en $B E P$ ne vaut que si l'élève trouve une place dans le LP de son choix et dans la section désirée. II n'est donc pas impossible qu'au mois de septembre des élèves n'ayant pu s'inscrire en LP reviennent au collège redoubler.

Au total combien d'élèves traversent sans embûche le collège à partir de la $5^{e}$ ? Sur les 1516 élèves qui constituent la base de l'enquête, seuls 596 arrivent en seconde en 3 ans, soit près de $40 \%(39,5 \%)$. Ils sont un peu plus nombreux à avoir réussi le brevet des collèges (42,5\%). La sélection est donc considérable et elle a eu lieu, dans les mêmes proportions, en fin de $5^{e}$ et en fin de $3^{e}$. Au total, 920 enfants ont quitté la cohorte entre la $5^{\mathrm{e}}$ et la fin de la $3^{\mathrm{e}}$. La classe de $5^{\mathrm{e}}$ est responsable de $40 \%$ des pertes, et la classe de $3^{e}$ de $41 \%$ de l'échec (14). Le taux d'échec, presque $60 \%$ de l'ensemble de la cohorte, est très important et plus élevé que la moyenne nationale. En effet, depuis le début des années 80 , ce sont plus de la moitié des élèves de $6^{\mathrm{e}}$ qui atteignent la seconde (15). Mais il faut tenir compte ici d'une divergence d'approche. Les recherches nationales auxquelles nous nous référons calculent les taux d'accès de la $6^{\mathrm{e}}$ à la seconde "quels que soient le cheminement suivi et le temps mis pour y parvenir". Ainsi sont pris en compte les redoublants de chaque niveau. Nos données doivent être interprétées avec prudence, puisqu'elles ne signifient pas que sur 100 élèves entrés en $6^{e}$ entre 1988 et 1989, seuls 39 d'entre eux atteindront la classe de seconde. Nous avons abandonné en cours de route les redoublants de $5^{\mathrm{e}}$, de $4^{e}$ et de $3^{e}$. Ce taux mesure donc en réalité la capacité des établissements à amener les élèves de la $5^{\mathrm{e}}$ à la $3^{\mathrm{e}}$ en temps " normal ", c'est-à-dire en 3 ans.

\section{Des inégalités persistantes}

En $3^{e}$, les filles restent avantagées. À note équivalente, elles passent plus en seconde que les garçons $(67 \% / 61 \%)$ et partent moins souvent en BEP. On retrouve donc ici, une nouvelle fois, la différence de traitement entre filles et garçons pour l'orientation. Mais il faut noter que si la $4^{e}$ technologique est quasi exclusivement masculine, le LP s'ouvre davantage aux filles. Ceci tient à la nature des filières proposées en LP. Alors que la $4^{e}$ technologique est uniformisée, les BEP regroupent de nombreuses sections fortement différenciées sexuellement (16).

La sélection en fin de $3^{\theta}$ selon les sexes rend la lecture des données plus complexe. Du fait de la sélection subie en fin de $5^{\mathrm{e}}$, les garçons se situent aux deux extrêmes de la notation en $3^{e}$; ce sont les garçons qui obtiennent les moins bonnes notes et ceux qui réussissent le mieux. Mais bien qu'ils obtiennent des notes meilleures que les filles, ils passent moins en seconde et partent plus souvent en BEP. La sélection en fonction du sexe demeure donc en fin de $3^{e}$, bien qu'elle soit plus faible qu'en $5^{e}$. Elle conduit à favoriser les filles sur l'en- 
semble de la cohorte. Un tiers seulement des garçons arrive à parcourir les 3 années du collège sans encombre ( $34 \% / 44,5 \%$ pour les filles).

Les enfants de cadres supérieurs et d'ouvriers se situent aux deux points opposés de la sélection: les premiers passent plus souvent que la moyenne en seconde (87\%) et lorsqu'ils échouent, ils redoublent presque autant qu'ils partent en LP $(5 \% ; 7,5 \%)$. Pour les enfants d'ouvriers, la sélection est beaucoup plus sévère: moins de la moitié d'entre eux arrivent en seconde (44\%), et leurs chances d'aller en LP sont tout aussi importantes (43\%) (17). Quand les élèves sont moyens (entre 8 et 11 de moyenne générale) les enfants de cadres supérieurs passent en seconde ou redoublent alors que les enfants d'ouvriers partent en BEP.

Tout au long de leur scolarité, les enfants des milieux populaires sont donc davantage sanctionnés que tous les autres. Ils ont près de trois fois moins de chances que les enfants de cadres supérieurs d'atteindre la seconde après 3 années de collège $(23 \% / 62,5 \%)$. Les enfants d'origine populaire auront donc subi deux fois une très forte défection, en $5^{\mathrm{e}}$ et en $3^{\mathrm{e}}$. A l'inverse, les enfants de cadres supérieurs n'ont presque jamais connu l'échec (18). Leurs chances de passer sont toujours supérieures à tous les autres, ils s'en sortent même mieux que les élèves qui n'ont jamais pris de retard : quand ils échouent, ils ne sont pratiquement jamais " orientés " vers le professionnel (19).

Au collège, les élèves les plus âgés ont une probabilité extrêmement faible de passer en seconde $(14,5 \%)$; ils ont, au contraire, de fortes chances de finir leurs études secondaires dans un LP $(83,5 \%)$. La sélection est un peu moins forte pour les élèves en retard d'une année jusqu'en $5^{\mathrm{e}}$, mais ils ont moins de chances d'aller en seconde (34\%) que de quitter l'enseignement général (52\%) ou de redoubler. Les élèves "à l'heure " ont, pour leur part, la quasi-assurance de prolonger leurs études dans un lycée classique ou polyvalent $(81,5 \%)$. Les écarts entre les élèves deviennent donc considérables en $3^{\mathrm{e}}$. La sélection $y$ est beaucoup plus importante qu'en $5^{e}$ où les enfants très en retard avaient encore presque $50 \%$ de chances de passer. Cette orientation en fonction de l'âge souligne le caractère discriminant de l'enseignement professionnel. Les futures classes de BEP sont ainsi composées de plus des 2/3 d'enfants ayant connu des échecs scolaires
(76\%) alors que, dans le même temps, les classes de lycée accueillent plus de $85 \%$ d'élèves qui n'ont jamais connu d'accrocs majeurs.

La projection sur l'ensemble de la cohorte met encore plus en lumière la différence de traitement à laquelle les élèves ont à faire face. Les élèves " à l'heure " survivent plus que la moyenne $(59,4 \%)$ alors que pour les autres, les chances d'effectuer le parcours en 3 ans sont dérisoires (18\% et $5 \%$ respectivement selon les années de retard).

\section{Disparité selon les établissements}

Comme en fin de $5^{e}$, la sélection en fin de $3^{e}$ connaît de fortes variations selon les établissements. Si $64 \%$ des collégiens passent en seconde, selon les collèges cette probabilité varie entre $21,5 \%$ et $78 \%$. Pour l'orientation en LP, les taux oscillent entre $15 \%$ et $67,5 \%$. Enfin, les chances d'atteindre la classe de seconde après 3 années d'études au collège varient, selon les établissements, entre $10,5 \%$ et $54 \%$, pour une moyenne de $39,5 \%$. Si ces données doivent être interprétées avec prudence, compte tenu de la faiblesse des effectifs dans certains établissements, elles indiquent malgré tout une extrême disparité des parcours des élèves selon leur contexte de scolarisation.

\section{Des logiques disparates}

Comment s'articulent les orientations de fin de $5^{e}$ et de fin de $3^{e}$ ? II n'existe pas, a priori, de lien entre les deux niveaux d'études, et tous les cas de figure peuvent se présenter. Une forte sélection en fin de $5^{e}$ ne met pas les élèves à l'abri d'une nouvelle sanction en fin de $3^{e}$ mais, à l'inverse, l'absence de sélection en fin de $5^{e}$, ne présage en rien de l'avenir des élèves. Le graphe cidessous met en évidence, d'une part, l'absence de relation qui existe entre les deux paliers d'orientation ( $R=0,25$, et $T=0,84$, la corrélation est donc non significative, même si la pente est positive) et, d'autre part, la complexité qui en découle. La sélection s'exerce à plusieurs niveaux : elle peut avoir lieu à chaque moment de l'orientation, ou n'avoir lieu qu'une fois ou encore, être faible à chaque palier.

La comparaison entre deux établissements (collèges 2 et 3 ) qui appliquent une sélection à chaque palier, montre qu'il existe des différences. Le premier collège est, les deux fois, très sélectif alors que le collège 3 , tout en demeurant plus 
Graphique 2. - Taux de passage en $2^{\text {de }}$ par collège par rapport aux taux de passage en $4^{\circ}$

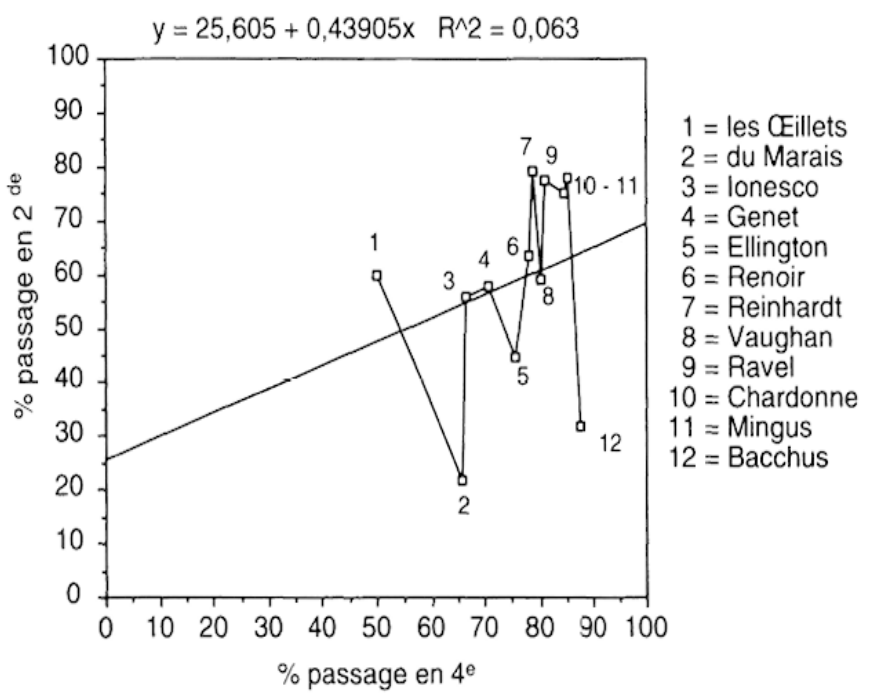

sélectif que bien d'autres établissements, applique une sanction plus " douce" en fin de $3^{e}$. Dans le premier cas, la sélection se renforce et se durcit au fur et à mesure alors que, dans le deuxième cas, elle va en s'atténuant. Les 12 collèges retenus dans cette étude illustrent les 4 cas de figures qui peuvent exister. Le tableau 2, ci-après, synthétise la répartition des établissements selon la manière dont ils pratiquent et articulent la sélection aux deux carrefours du collège.

Mais qu'en est-il de l'effet établissement ? Quand on oppose les collèges en fonction de leur sélectivité à chaque palier, force est de constater que ce sont les établissements les moins favorisés qui pénalisent le plus leurs élèves. L'hypothèse d'un effet d'établissement apparaît précaire et ne s'appliquerait qu'à un seul cas, le collège J. Renoir. II

Tableau 2. - Sélection scolaire en $5^{\circ}$ et en $3^{\circ}$

\begin{tabular}{|c|c|c|c|}
\hline \multicolumn{2}{|c|}{} & \multicolumn{2}{c|}{$5^{\mathrm{e}}$} \\
\hline \multirow{2}{*}{} & + & - \\
\hline \multirow{3}{*}{$3^{\mathrm{e}}$} & + & $\begin{array}{c}\text { Le Marais } \\
\text { lonesco }\end{array}$ & $\begin{array}{c}\text { Bacchus } \\
\text { Ellington }\end{array}$ \\
\cline { 2 - 4 } & & $\begin{array}{c}\text { Les CEillets } \\
\text { Vaughan } \\
\text { Genet }\end{array}$ & $\begin{array}{c}\text { Renoir } \\
\text { Reinhardt } \\
\text { Raval } \\
\text { Chardonne } \\
\text { Mingus }\end{array}$ \\
\hline
\end{tabular}

est, en effet, le seul collège populaire qui, par rapport à la moyenne, pénalise faiblement les élèves en fin de $5^{e}$ et en fin de $3^{e}$. Mais ce raisonnement ne correspond pas exactement avec la définition que nous avons retenue de l'effet d'établissement, à savoir que la sélectivité d'un établissement doit être interprétée de manière relative par rapport à un établissement socialement comparable. Certes, les établissements socialement favorisés ne se retrouvent jamais dans la catégorie des collèges qui sanctionnent fortement leurs élèves. Mais on peut noter par ailleurs que, dans les autres établissements, il n'existe pas d'unité dans la carrière scolaire des élèves. Ainsi, les trois collèges les plus populaires (le Marais, les CEillets et Bacchus) ne pratiquent pas la même politique. Au sein d'une même catégorie d'établissements, il existe donc des divergences non négligeables.

Cette première présentation ne permet pas d'affirmer l'existence d'un effet d'établissement mais la combinaison est trop complexe pour que seul un effet de composition puisse rendre compte de l'orientation des collèges. La problématique de l'effet d'établissement ne consiste pas à distinguer les collèges selon deux catégories - sélectifs/nonsélectifs- mais à saisir, à population équivalente, les variations de l'orientation entre les établissements. Or l'analyse de régression entreprise en fin de $3^{e}$ tend à montrer qu'il existe un effet propre de l'établissement (20). Quelles que soient, par ailleurs, les caractéristiques individuelles des élèves, le collège a un effet propre sur la probabilité de passer en seconde. Ainsi, quand le collège est considéré comme une variable explicative, il est possible de rejeter l'hypothèse d'indépendance entre le passage en seconde et l'établissement, toutes choses égales par ailleurs (sexe, âge, CSP, notes, année d'entrée dans l'établissement et statut scolaire (21)). À population équivalente, les élèves ne connaissent pas la même orientation. Les collèges ont des effets propres qui ne peuvent s'expliquer uniquement par la population accueillie.

L'effet d'établissement se traduit donc de la manière suivante: si tous les collèges accueillaient la même population, sept d'entre eux ne pratiqueraient pas la même orientation. Ainsi, la similitude des établissements est loin de garantir une équivalence des résultats. II faut prendre en compte d'autres facteurs que le recrutement social : la politique éducative et pédagogique, le secteur géographique ou le type d'enseignants, 
par exemple. L'effet d'établissement ne conduit évidemment pas à rejeter la relation entre composition sociale et orientation des collèges, mais il existe des écarts à la moyenne suffisamment importants pour que nous puissions nous interroger sur la manière dont les acteurs sociaux construisent leur établissement afin que celui-ci produise un effet propre, qui n'est pas nécessairement visé par les individus.

Le graphe ci-dessous, qui distingue les établissements selon les chances de survie des élèves, illustre ce propos. II existe un effet établissement dans la mesure où, à profil équivalent, les chances de passer en seconde varient significativement selon les collèges. La relation est significative $(R=0,729$ et $T=3,35$, significatif à 0,05$)$. La tendance générale dessine donc une relation forte et positive entre la composition sociale des établissements et le taux de passage en seconde. Mais, autour de cette tendance, la variance reste grande et les écarts d'un établissement à l'autre sont loin d'être négligeables. Ainsi, les collèges du Marais, Bacchus, D. Ellington et J. Chardonne (collèges 2, 3, 8 et 11) s'écartent notablement de cette tendance générale, de même que les collèges J. Renoir, D. Reinhardt et M. Ravel (collèges 4,9 et 10). Les premiers sont plus sélectifs que prévus, alors que les seconds le sont moins. Les

Graphique 4. - Taux de passage en $2^{\text {de }}$ par collège par rapport à la totalité inscrits en $5^{\circ}$ en fonction d'enfants de cadres supérieurs $y=22,376+0,86297 x \quad R^{\wedge} 2=0,531$

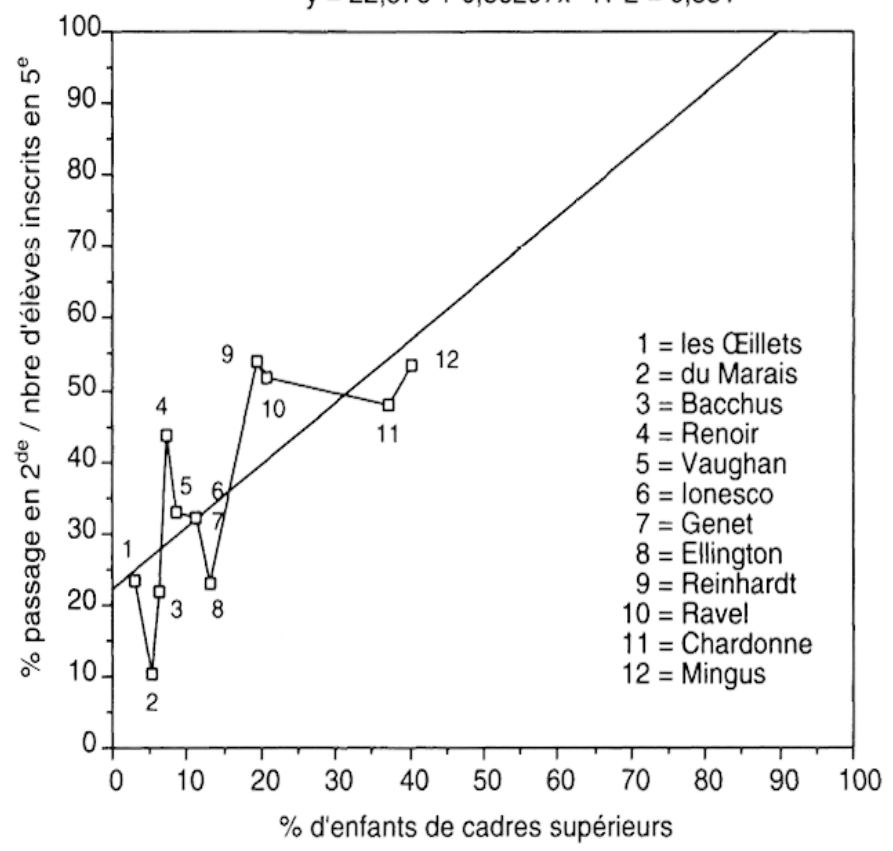

autres établissements se situent dans la moyenne. On ne peut parler d'effet établissement que pour 7 des 12 collèges retenus dans cette étude.

\section{LES POLITIQUES D'ÉTABLISSEMENTS}

Comment expliquer ces différences d'orientation entre des établissements au recrutement proche? À partir des entretiens avec les différents acteurs des collèges et des observations de la vie des établissements, nous avons reconstitué les " politiques" des collèges, c'est-à-dire l'existence ou l'absence de logique d'action. Quatre principales dimensions peuvent en rendre compte : le rapport à l'environnement, la politique de l'équipe de direction, la cohésion professionnelle au sein de l'établissement et les actions mises en place. Ces dimensions seront résumées à des valeurs simples et dichotomiques. Après une brève présentation de ces dimensions, nous essayerons de voir comment elles s'articulent en nous demandant si les établissements réussissent ou "échouent " pour les mêmes raisons (22).

\section{Les dimensions des politiques d'établissements}

\section{Le rapport à l'environnement}

Deux indicateurs permettent d'en rendre compte ; la place de l'établissement sur le marché scolaire et la représentation de l'environnement par les acteurs. Le premier indicateur est apprécié à partir des demandes de dérogation pour l'inscription en $6^{e}$ et de l'aire de recrutement des élèves. Le deuxième prend en compte la perception de l'environnement par le collège. Selon cette représentation le contexte peut devenir une ressource pour l'établissement ou, au contraire, apparaître comme un obstacle et nuire au collège. Les établissements se situent sur le versant positif de cette dimension s'ils sont attractifs (23) et ouverts sur leur environnement, ils se situent sur le versant négatif s'ils perdent chaque année des élèves et se replient sur eux-mêmes.

\section{Politique de l'équipe de direction}

Les travaux portants sur l'école "efficace " ou sur l'effet établissement insistent particulièrement sur le rôle de la direction, bien qu'ils ne leur accordent pas tous la même importance (24). Cette dimension est évaluée à partir de la distri- 
bution des fonctions au sein de l'équipe de direction et des relations entre l'administration et les professeurs. Le premier indicateur apprécie la répartition des rôles entre le principal et son adjoint et la cohésion qui existe au sein de la direction (25). Le deuxième met en évidence les relations entre les enseignants et l'administration et plus particulièrement le décloisonnement entre les tâches pédagogiques et les tâches administratives. Les établissements se situent sur le versant positif de cette dimension si le principal et l'adjoint collaborent et si l'ensemble des acteurs reconnaît à la direction une légitimité à intervenir dans le domaine pédagogique. Au contraire, ils se situent sur le versant négatif de cette dimension quand l'équipe de direction ne travaille pas de concert et quand les problèmes pédagogiques restent le domaine exclusif des enseignants.

\section{Nature de la cohésion professionnelle}

La cohésion professionnelle s'appuie sur plusieurs indicateurs. Le premier s'attache plus particulièrement aux relations entre les professeurs à travers la convivialité du collège et l'ambiance de la salle des professeurs (26). Le deuxième indicateur prend en compte les relations de travail; notamment la définition du métier d'enseignant et de l'acte pédagogique, la définition donnée par chacun des objectifs à atteindre. Enfin, il faut tenir compte de la perception du changement de ces deux dernières décennies. Selon les établissements et les individus, les discours sont plus ou moins empreints de nostalgie. Les établissements se placent sur le versant positif de cette dimension quand il n'existe pas de conflit majeur entre les professeurs, quand ils partagent les mêmes objectifs et portent un regard similaire sur l'évolution de l'école ces dernières années. À l'inverse, ils se situent sur le versant négatif quand les rela- tions entre les enseignants sont dominées par des conflits empêchant tout accord sur les objectifs à atteindre et quand ils n'ont pas ressenti de la même manière les transformations de l'école.

\section{Action et mobilisation des acteurs}

Les projets d'établissement sont souvent trop imprécis sur les objectifs et, surtout, ils prennent plus souvent la forme d'un exercice routinier réalisé par le chef d'établissement pour permettre de mesurer les orientations des collèges (27). Pour évaluer la mobilisation des enseignants, il faut tenir compte de leurs capacités à confronter leurs pratiques, leurs méthodes pédagogiques et à tenter d'élaborer ensemble des projets visant à atteindre des objectifs communs. Les actions mises en place sont appréciées en fonction de leur ampleur et, surtout, de leur cohérence et de leur articulation. Les établissements se situent sur le versant positif de cette dimension quand il existe une mobilisation collective autour d'actions précises et solidaires. En revanche, ils se situent sur le versant négatif de cette dimension quand les enseignants travaillent seuls et de manières individuelles et lorsque les actions existantes restent cloisonnées.

\section{Effet établissement et articulation des dimensions}

La comparaison des six établissements se démarquant de la moyenne quant à leur sélectivité globale (taux de passage en seconde par rapport aux inscrits en $5^{\mathrm{e}}$ trois ans plus tôt) permet de souligner les homologies et les différences entre collèges. Ainsi nous pouvons présenter à l'aide du tableau ci-dessous comment se situent les six collèges sur les quatre dimensions.

Tableau 3. - Les dimensions des politiques d'établissement

\begin{tabular}{|c|c|c|c|c|c|c|c|c|}
\hline & \multicolumn{2}{|c|}{ Contexte } & \multicolumn{2}{c|}{ Administration } & \multicolumn{2}{c|}{ Cohésion } & \multicolumn{2}{c|}{ Mobilisation } \\
\hline & - & + & - & + & - & + & - & + \\
\hline $\begin{array}{c}\text { Collège peu } \\
\text { sélectif }\end{array}$ & $\begin{array}{c}\text { Renoir } \\
\text { Reinhardt } \\
\text { Ravel }\end{array}$ & & $\begin{array}{c}\text { Renoir } \\
\text { Reinhardt } \\
\text { Ravel }\end{array}$ & $\begin{array}{c}\text { Renoir } \\
\text { Reinhardt } \\
\text { Ravel }\end{array}$ \\
\hline $\begin{array}{c}\text { Collège } \\
\text { sélectif }\end{array}$ & $\begin{array}{c}\text { Marais } \\
\text { Ellington } \\
\text { Ravel }\end{array}$ & Marais & Ellington & Marais & $\begin{array}{c}\text { Marais } \\
\text { Ellington } \\
\text { Chardonne }\end{array}$ & \\
\hline
\end{tabular}


La comparaison entre les collèges " peu sélectifs" et les établissements "sélectifs" révèle une grande différence. Les trois premiers établissements se regroupent sur toutes les dimensions et ils se situent toujours sur le versant positif. Les collèges "peu sélectifs" bénéficient d'un contexte favorable et ils sont en position de leader sur le "marché " scolaire, ce qui leur permet de recruter hors de leur secteur d'origine et de maintenir leur effectif chaque année. Ils n'ont pas à faire au même type de direction mais aucun des trois ne connaît de conflit au sein de l'équipe de direction et entre les professeurs et l'administration. Les enseignants entretiennent plutôt de bonnes relations et s'ils ne partagent pas tous les mêmes objectifs, ils reconnaissent la légitimité des orientations de leurs collègues ou ne cherchent pas à s'y opposer. Enfin, il existe un degré de mobilisation suffisamment important pour que l'établissement entreprenne des actions de plus ou moins grandes envergures et les exploite. Les actions participent pleinement à la construction de l'identité de l'établissement, elles consolident la réputation des collèges. La nature de ces actions varie selon les collèges, elles permettent aux enseignants de participer à la vie de l'établissement et de briser l'isolement, soit par un engagement autour d'activités éducatives, soit par un engagement autour d'une politique pédagogique.

À l'inverse, pour les collèges " sélectifs " deux phénomènes apparaissent. D'une part, ils sont plus hétérogènes que dans le cas précédent, ils ont des profils différents. D'autre part, les quatre dimensions retenues ne s'harmonisent pas. Ils peuvent être parfois sur le versant positif d'une dimension. Ainsi, ces collèges sont "sélectifs" pour des raisons différentes. Le Marais est victime de son contexte et ne réussit pas à sortir de la problématique de la "reprise en main". Toute l'activité du collège est tournée autour des problèmes de discipline. À J. Chardonne c'est le refus par des enseignants de la politique de la direction et du "style" du principal, jugé trop médiatique, qui entrave toute réflexion collective. Les professeurs n'accordent qu'une faible légitimité au directeur. Enfin, D. Ellington cumule les handicaps, dont les deux principaux proviennent des conflits entre les professeurs et de l'impossibilité du directeur à s'imposer.

Comment jouent ces différentes dimensions? Comment permettent-elles d'expliquer l'effet éta- blissement ? Aucune de ces quatre dimensions ne peut, à elle seule, rendre compte de l'effet établissement. II s'agit d'une combinaison complexe de différents facteurs qui s'articulent de manière particulière dans chaque collège.

Le contexte apparaît à bien des égards un élément essentiel pour comprendre les logiques d'établissements. Parce que les collèges et les lycées se situent dans un "marché " scolaire, la réputation de l'établissement devient un enjeu central. Ils doivent gérer et préserver leur " image " auprès des familles et de leurs partenaires. Ils héritent d'une réputation fondée principalement sur l'ancien statut de l'établissement et tentent de la modeler, c'est pourquoi l'ouverture sur l'extérieur et les activités éducatives tiennent une place si déterminante. Le contexte peut donc être un des stimulants de la politique de l'établissement. En revanche, il n'est pas une condition suffisante pour expliquer la sélection des élèves. II devient un obstacle quasi insurmontable quand l'établissement est aussi faible sur les autres dimensions, comme c'est le cas au Marais.

Le rôle de l'équipe de direction est incontestable dans l'élaboration d'une politique d'établissement. Elle est souvent le seul acteur possédant un regard d'ensemble sur l'établissement. Elle peut donc proposer le bilan des activités entreprises et, éventuellement, des solutions. La gestion de la vie quotidienne de l'établissement et l'organisation des emplois du temps peuvent s'avérer essentiels dans l'élaboration de la politique d'établissement. Mais le rôle et la capacité d'action de l'équipe de direction ne se comprennent que dans les relations complexes avec les différents acteurs. Les nominations sont indépendantes de la volonté des établissements. Les équipes de direction se composent d'hommes et de femmes qui n'ont pas toujours les mêmes conceptions de leur métier et de l'école en général. Le rôle de l'équipe de direction tient moins au style du chef d'établissement qu'à la légitimité qu'il tire de son action et des attentes de l'ensemble de l'établissement à son égard. Aucun style de direction ne semble indiquer a priori quelle sera la politique de l'établissement, ni ses conséquences sur la vie du collège ou du lycée. La direction ne devient centrale que lorsqu'elle trouve un point d'appui soit au sein de l'équipe d'encadrement (adjoint, CPE, etc.) soit parmi le corps professoral. La position de l'équipe de direction est d'autant plus fragile qu'elle reste souvent moins longtemps en 
place que les enseignants. Nous sommes donc tenté de tempérer le poids accordé à l'équipe de direction dans l'effet établissement. Faire du chef d'établissement un "manager " à l'image d'un chef d'entreprise, comme cela a été souvent le cas cette dernière décennie, paraît peu crédible compte tenu de la résistance du corps enseignant à ce type de référence et de sa volonté farouche de préserver son domaine réservé - la pédagogie - et parce que le directeur n'a pas le pouvoir de s'imposer dans ce domaine.

La cohésion d'une équipe pédagogique est par définition fragile. Les collèges accueillent des enseignants aux parcours divers, ayant des formations différentes et des histoires professionnelles variées. Ils n'ont plus ni la même histoire politique et syndicale ni la même représentation du métier. En revanche, ce qui continue à rassembler les professeurs c'est la référence à la discipline enseignée. Quels que soient leur âge et leur expérience, les professeurs s'identifient à leur discipline. L'exercice pédagogique demeure encore très largement individuel à l'exception notable de celui des professeurs de technologie et d'éducation physique et sportive. Même dans les établissements où il existe une forte cohésion de l'équipe enseignante, les professeurs tiennent à préserver leur indépendance. Ils veulent être maîtres des textes sur lesquels ils s'appuient, des outils qu'ils utilisent et de la progression du programme. La cohésion entre les enseignants est un facteur nécessaire à la mobilisation mais il n'est pas suffisant.

Le degré de mobilisation et la nature des actions existantes démarquent nettement les deux types d'établissements. Sur cette dimension, les collèges "peu sélectifs" se regroupent sur le versant positif bien qu'il existe des différences. Le collège $M$. Ravel se caractérise par une forte cohésion professionnelle et une capacité de mobilisation importante. Les enseignants suivent la politique définie par le principal et s'engagent dans des actions collectives qui leur demandent de confronter leur pratique et les obligent à pratiquer une concertation disciplinaire. La politique de l'établissement se traduit par l'harmonisation de la progression des cours, la réintroduction des devoirs surveillés par niveau d'étude et l'élaboration de nombreux PAE (28) d'envergure. $\dot{A}$ J. Renoir, la cohésion professionnelle est plus fragile, ce qui conduit à une mobilisation éclatée. Quelques enseignants, emmenés par la CPE, la documentaliste et un professeur, organisent les activités éducatives et tentent de rénover les actions pédagogiques. La résistance de certains enseignants rend parfois difficile l'harmonisation des initiatives et les activités se succèdent sans toujours s'articuler. D. Reinhardt représente un cas intermédiaire. Le principal adjoint et la CPE organisent depuis de nombreuses années les activités pédagogiques et éducatives. Une partie des enseignants suit ces initiatives et accepte de travailler collectivement. Une autre partie demeure plus réticente et reste en retrait. Dans les trois collèges, l'effet établissement, entendu ici comme la capacité de passer d'une norme centrée sur la classe à une norme d'ensemble, tient à la rencontre entre un corps enseignant réceptif aux problèmes qui l'entourent et désireux d'y remédier, et un groupe de personnes capables de stimuler l'ensemble.

Les collèges "sélectifs" se caractérisent par l'éclatement des logiques. Le collège du Marais consacre l'essentiel de son énergie à un rétablissement de la discipline et se donne comme objectif de "re-socialiser" les élèves. Les actions pédagogiques apparaissent en demi-teinte et, quand elles existent, se révèlent faibles. Bien que le collège soit en ZEP, les échanges avec l'environnement sont rares et l'établissement cherche plutôt à se protéger. L'absence de mobilisation est tout aussi frappante à D. Ellington. Les enseignants déplorent l'inertie du collège mais ils ne réussissent pas à élaborer ensemble une norme d'établissement parce qu'ils s'opposent sur les moyens et les objectifs à atteindre. Les conflits statutaires et d'ancienneté dominent les relations entre les professeurs empêchant toute réflexion d'ensemble. Enfin, J. Chardonne offre deux visages contradictoires. II est très mobilisé autour des activités éducatives, multipliant les voyages à l'étranger et les échanges avec les partenaires extérieurs. Mais il n'existe pas d'engagement dans une politique pédagogique parce que les professeurs n'en ressentent pas la nécessité. Ils visent "l'excellence scolaire " et, compte tenu de la population accueillie, principalement des enfants issus des milieux favorisés, cet objectif n'exige pas une mobilisation collective. La majorité des enseignants tient à préserver son indépendance et défend une conception traditionnelle du métier.

Les raisons qui poussent les enseignants à s'engager dans la vie de l'établissement restent très 
aléatoires et il n'existe, semble-t-il, aucun modèle capable d'en rendre compte. Elles sont dues au hasard des nominations et des rencontres entre des enseignants partageant les mêmes espoirs ou les mêmes orientations politiques et syndicales. La participation à des stages ou le refus de sombrer dans le fatalisme et la nostalgie sont aussi des raisons avancées par les acteurs pour expliquer leur volonté d'action. II faut aussi tenir compte du public de l'établissement. II est probable que les enseignants ressentent la nécessité de s'engager dans une politique d'ensemble quand ils ne sont pas pris par l'urgence. Autrement dit, parmi les douze collèges de cette étude, ce sont les établissements où l'échec scolaire est perçu comme un phénomène encore marginal qui réussissent le plus à mettre en place des actions. À chaque fois, enseignants et équipes de direction rappellent qu'ils ont voulu réagir face au petit nombre d'élèves en difficulté. Dans les établissements confrontés à un plus grand nombre d'enfants en difficulté scolaire, la mobilisation apparaît toujours plus fragile et plus faible. L'hétérogénéité des élèves et des parcours des professeurs devient l'obstacle à une prise de décision collective. Mais, les établissements les plus prestigieux se heurtent au même type de difficulté. Dans ce cas, l'établissement défend un enseignement traditionnel et refuse souvent le changement de comportement des élèves. Ainsi, le profil des établissements les plus mobilisés correspond à des collèges d'implantation assez récente accueillant une population composée, pour partie, d'enfants issus des petites classes moyenne (29). Ils ne sont ni submergés par des difficultés ni prisonniers d'un passé et d'une tradition.

Enfin, il convient de tenir compte de l'histoire récente des collèges pour comprendre les obstacles à la mobilisation. II est en effet frappant de constater le sentiment de désillusion et d'impuissance qui règne dans certains collèges. Depuis le début des années 80 , quelquefois avant, les collèges tentent diverses expériences. Les enseignants cherchent à travers les stages et les nouvelles techniques pédagogiques à limiter l'échec scolaire. Or, il n'est pas rare qu'ils aient aujourd'hui le sentiment d'avoir "tout essayé ". Ils ne croient plus aux grandes réformes et se méfient de la décentralisation. La concertation est souvent perçue comme une surcharge de travail et non comme un remède ou un outil efficace. Les enseignants peuvent se sentir d'autant plus démunis que la pression des familles s'accroît. On demande aujourd'hui à l'école à la fois de former des individus et de réduire le chômage (30). Les professeurs, pour se protéger, se replient sur ce qu'ils "savent faire" : enseigner une discipline. Ce sentiment est diversement partagé par les professeurs. Selon les collèges, il est plus ou moins dominant mais tout se passe comme si enseignants et équipe de direction voulaient " marquer une pause".

\section{Politique d'établissement et orientation scolaire}

Qu'en est-il de la relation entre mobilisation des établissements et les résultats scolaires des élèves? Les trois collèges "peu sélectifs", par rapport à la moyenne, sont aussi les trois établissements qui présentent le plus haut degré de cohésion et de mobilisation. À l'intérieur de ce groupe d'établissements, peut-on établir une hiérarchie ? Si l'on se place du point de vue de la cohésion sociale au sein de l'établissement et du degré de mobilisation, $M$. Ravel est le collège le plus "dynamique " et le plus cohérent, puis viennent D. Reinhardt et J. Renoir. Or, la sélectivité de M. Ravel demeure plus forte qu'à $D$. Reinhardt. En effet, pour 100 élèves inscrits en $5^{e}$ en 1990 , 54,2 atteignent la classe de seconde 3 années plus tard à D. Reinhardt et 51,6 à M. Ravel. Le taux de "survie" pour J. Renoir est de 43,7. Cette différence, entre Ravel et Reinhardt, n'est pas statistiquement significative mais elle nous oblige à interpréter avec prudence la relation entre mobilisation et orientation scolaire.

La mobilisation affecte la conception du métier d'enseignant. À l'écoute des différents entretiens, il apparaît que la mobilisation réduit la distance entre le "statut" et le " métier ". En effet, tout se passe comme si la "conscience malheureuse" induite par la référence au statut avec toutes les contraintes que cela suppose s'effaçait devant le sentiment de liberté d'action qu'offre le métier. À D. Reinhardt ou à J. Renoir, les enseignants se sentent tiraillés entre leurs convictions et les pesanteurs auxquels ils doivent faire face. Au contraire, à $M$. Ravel les enseignants réussissent à dépasser ce clivage. Métier et statut ne s'opposent plus et semblent faire un tout. L'opposition entre le versant éducatif des activités et les nécessités pédagogiques des programmes s'atténue, et les professeurs déclarent pouvoir instruire et éduquer les jeunes. Ainsi, de ce point de vue, 
la mobilisation des acteurs peut être comprise comme un moyen de réduire le sentiment de crise et de malaise qui parcourt depuis plusieurs années le milieu enseignant. Ce sont d'ailleurs les collèges où le corps professoral connaît la plus faible cohésion qui développent le plus souvent le discours sur la "crise de l'école".

Le dernier aspect de l'effet établissement concerne la relation entre la mobilisation des acteurs et la réduction des inégalités. Les collèges les plus engagés dans une politique d'ensemble deviennent-ils plus équitables? Selon l'indicateur retenu, les réponses varient.

Les établissements les plus mobilisés se révèlent être aussi socialement les plus équitables. En effet, dans les trois collèges " peu sélectifs ", où existe une politique d'établissement, les enfants d'ouvriers voient leurs chances d'accéder en seconde trois ans après la $5^{\mathrm{e}}$ croître. Les écarts entre enfants d'ouvriers et enfants des milieux favorisés se réduisent par rapport à la moyenne (31). On peut même remarquer que $M$. Ravel, qui offre le plus de chances aux enfants d'ouvriers d'accéder au lycée, connaît la plus forte cohésion et la plus forte mobilisation. Mais les données doivent être interprétées avec prudence compte tenu de la faiblesse des effectifs. Ce qui distingue essentiellement ces trois collèges des établissements "sélectifs" et sans politique globale, c'est leur capacité d'accroître les chances de réussite des enfants d'ouvriers et des enfants de cadres. Dans les collèges "sélectifs " les écarts se réduisent mais au détriment des enfants des milieux favorisés. Ainsi à J. Chardonne les enfants d'ouvriers réussissent conformément à la moyenne alors que les enfants de cadres passent moins souvent en seconde. Au Marais ce sont tous les élèves qui connaissent un échec massif. Ainsi, la construction d'une norme d'établissement semble être un facteur de réduction des inégalités sociales.

Le lien entre la politique d'établissement et l'âge des élèves apparaît plus aléatoire. II n'existe plus de relation stable. Comme avec l'origine sociale, les collèges "sélectifs" réduisent les écarts entre les jeunes n'ayant pas accumulé de retard et les élèves ayant un an de retard, mais cette réduction se fait au détriment des premiers. En revanche, dans les établissements "peu sélectifs " la tendance semble être à l'aggravation. Par rapport à la moyenne les élèves en retard réussissent moins bien. M. Ravel devient l'établis- sement le plus pénalisant ainsi que J. Renoir alors que $D$. Reinhardt réduit légèrement les écarts (32).

\section{$\stackrel{*}{* *}$}

L'effet établissement entendu comme la capacité d'un établissement de construire une norme d'ensemble rend compte de plusieurs phénomènes. La sélectivité des collèges les plus mobilisés ne s'explique pas totalement à partir de leur recrutement social. Ils réussissent à emmener plus d'élèves en seconde en trois ans que des collèges semblables qui n'ont pas de politique d'ensemble. Cette politique permet aussi aux établissements d'atténuer partiellement les inégalités sociales. Les enfants de cadres réussissent mieux que tous les autres mais les enfants d'ouvriers voient leurs chances d'entrer au lycée augmenter par rapport aux autres collèges. En revanche, les élèves les plus âgés et ceux qui ont redoublé ne bénéficient pas ou très peu de "l'effet établissement". La mobilisation des acteurs ne permet donc pas de réduire l'échec scolaire, et les élèves en difficulté, au parcours chaotique, demeurent les plus pénalisés. L'effet établissement s'avère "efficace " pour les "bons" élèves, ceux qui n'ont jamais connu d'accrocs, quelle que soit leur origine sociale. II est beaucoup plus fragile et moins " efficace" pour les autres.

La mobilisation des acteurs ne semble pas toujours avoir un effet direct et précis sur l'orientation des élèves. En revanche, il semble incontestable que dans les collèges ayant le degré le plus élevé de cohésion, les enseignants vivent mieux leur métier, et les thèmes de la crise de l'école, du malaise enseignant et de l'hétérogénéité des élèves sont moins présents. La conscience malheureuse des professeurs, si souvent rencontrée dans les collèges, s'atténue dans ces établissements et les professeurs se sentent moins désarmés et abandonnés qu'ailleurs. Cette observation, pour banale qu'elle soit et malgré son caractère quasi tautologique, apparaît comme un facteur non négligeable, puisque ce sont aussi dans ces établissements que les adultes parlent le plus positivement des élèves et donnent l'impression que leur action fait encore sens.

Ainsi, l'effet établissement se révèle fragile et soumis aux impératifs contextuels et temporels, il ne permet certainement pas de rendre compte de la totalité des logiques d'orientation des établisse- 
ments ainsi que de la réussite des élèves. Malgré l'autonomie accordée aux établissements, les politiques d'établissement restent encore très informelles. Quand elles existent, elles permettent de faire de l'établissement un lieu de négociation dans lequel peuvent s'exprimer les conflits et les enjeux autour d'une définition de l'école. Elles permettent aussi aux professeurs de ne plus subir une situation mais au contraire de mieux supporter et, peut-être, de se dégager des contraintes administratives et bureaucratiques. Mais pour que les politiques d'établissement puissent perdurer il est nécessaire qu'elles se construisent sur le long terme et ne soient pas à la merci de l'instabilité du corps enseignant et de l'équipe administrative. C'est probablement un des obstacles majeurs à leur élaboration.

Olivier Cousin CNRS, CADIS, Université de Bordeaux II

\section{ANNEXE}

Tableau $4^{*}$ - Caractéristiques générales des établissements : effectifs de $5^{\bullet}$ et taux de passage en seconde par rapport au nombre d'élèves inscrits en $5^{e}$.

\begin{tabular}{|c|c|c|c|c|c|c|}
\hline Collèges & $\begin{array}{c}\text { Nombre } \\
\text { d'élèves }\end{array}$ & $\%$ ouvriers & $\begin{array}{c}\% \text { cadres } \\
\text { supérieurs }\end{array}$ & $\begin{array}{c}\text { Zones } \\
\text { géographiques }\end{array}$ & $\begin{array}{c}\text { Contexte } \\
\text { scolaire }\end{array}$ & $\begin{array}{c}\text { Tx de passage } \\
\text { en 2de/ } \\
\text { effectif de } 5^{\text {de }}\end{array}$ \\
\hline Bacchus & 32 & 15,5 & 0 & Rurale & Seul & 22 \\
\hline Les CEillets & 64 & 50 & 3 & Centre ville & Autre établ. & 23,5 \\
\hline Le Marais & 76 & 37 & 5,5 & Banlieue & Autre établ. & 10,5 \\
\hline J. Renoir & 183 & 16,5 & 7 & Banlieue & Autre établ. & 43,5 \\
\hline S. Vaughan & 70 & 20 & 8,5 & Banlieue & Autre établ. & 33 \\
\hline lonesco & 190 & 22 & 11 & Banlieue & Seul & 32 \\
\hline J. Genet & 225 & 34 & 11 & Banlieue & Autre établ. & 32,5 \\
\hline D. Ellington & 69 & 23 & 13 & Ville moyenne & Autre établ. & 23 \\
\hline M. Reinhardt & 120 & 7,5 & 19,5 & Banlieue & Autre établ. & 54 \\
\hline D. Ravel & 118 & 10 & 21 & Banlieue & Autre établ. & 51,5 \\
\hline J. Chardonne & 177 & 5 & 37,5 & Centre ville & Autre établ. & 48 \\
\hline C. Mingus & 192 & 3,5 & 40 & Centre ville & Autre établ. & 53 \\
\hline Total & 1516 & 18,5 & 18 & & & 39,5 \\
\hline
\end{tabular}

* L'ordre de présentation des collèges est établi à partir de leur recrutement social, allant de celui qui accueille la plus faible proportion d'enfants de cadres supérieurs à celui qui en reçoit le plus.

\section{BIBLIOGRAPHIE}

BALLION R. (1982). - Les consommateurs d'école. Paris : Stock.

BALLION R. (1992). - La bonne école. Paris: Hatier.

BALLION R. (1993). - Le lycée, une cité à construire. Paris : Hachette.

BAUDELOT C., ESTABLET R. (1992). - Allez les filles. Paris : Seuil.

BERTHELOT J.-M. (1993). - École, orientation, société. Paris : PUF.

CAHIERS FRANÇAIS (LES) (1991). - Le système éducatif. La Documentation française, $n^{\circ} 249$, janvierfévrier.
COUSIN O. (1993). - L'effet établissement. Construction d'une problématique. Revue française de sociologie, vol. $34, n^{\circ} 3$, juillet-septembre, p. 395-419.

COUSIN 0. (1994). - L'effet établissement, étude comparative de douze collèges. Thèse de doctorat de sociologie, Université de Bordeaux II, janvier 1994.

COUSIN O., GUILLEMET J.-P. (1992). - Variation des performances scolaires et effet d'établissement. Éducation et Formations, $n^{\circ} 31$, avril-juin, p. 23-30.

DEP (1992). - Repères et références statistiques sur les enseignements et la formation. Paris : Ministère de l'éducation nationale et de la culture, DEP. 
DEROUET J.-L. (1992). - École et justice. De l'égalité des chances aux compromis locaux? Paris: Ed. Métailé.

DUBET F., COUSIN O., GUILLEMET J.P. (1989). Mobilisation des établissements scolaires. Le cas des collèges. Revue française de sociologie, vol. XXX, n $n^{\circ}$ 2, p. 235-256.

DURU-BELLAT M. (1990). - L'école des filles. Paris : L'Harmattan.

DURU-BELLAT M., HENRIOT-VAN ZANTEN, A. (1992). - Sociologie de l'école. Paris : A. Colin.

DURU-BELLAT M., MINGAT A. (1993). - Pour une approche analytique du fonctionnement du système éducatif. Paris : PUF.

DURU-BELLAT M., JARROUSSE J.P., MINGAT A. (1993). - Les scolarités de la maternelle au lycée. Revue française de sociologie, vol. XXXIV, $n^{\circ} 1$, p. $43-60$

DURU M., MINGAT A. (1985). - De l'orientation en fin de cinquième au fonctionnement du collège. Tome 1: Évaluation de la procédure. Dijon: Cahiers de I'IREDU, $n^{\circ} 42$

DURU M., MINGAT A. (1988). - De l'orientation en fin de cinquième au fonctionnement du collège. Tome 2: Progression, notation, orientation, : l'impact du contexte de la scolarisation. Dijon: Cahiers de l'IREDU, $n^{\circ} 45$.
FELOUZIS G. (1994). - Le collège au quotidien. Paris : PUF

FRANÇOIS R., OBIN J.-P. (1991). - Audit des dispositifs concernant les projets d'établissement. Paris: Ministère de l'Éducation nationale, mai 1991, rapport ronéotypé.

GRISAY A. (1989). - Quels indicateurs d'efficacité pour les établissements scolaires? Étude d'un groupe contrasté de collèges "performants " et "peu performants". Liège : Université de Liège, Service de pédagogie expérimentale.

PATY D. (1981). - Douze collèges en France. Paris : La Documentation française.

OBIN J.-P. (1993). - La crise de l'organisation scolaire, Paris : Hachette.

OBIN J.-P., WEBER A. (1987). - Les projets d'établissement scolaire: de l'imaginaire à la réalité. Éducation permanente, $n^{\circ} 87$, p. 49-64.

Rapport de l'Inspection générale de l'Éducation nationale (1991). - Paris: La Documentation Française.

DUTERCQ Y. (1991). - Thé ou café ? Ou comment l'analyse de réseau peut aider à comprendre le fonctionnement d'un établissement scolaire. Revue française de pédagogie, $n^{\circ} 95$, avril-mai-juin.

\section{NOTES}

* Cet exposé s'appuie sur une recherche effectuée dans le cadre d'un doctorat en sociologie. $O$. Cousin, L'effet établissement, étude comparative de 12 collèges, Université de Bordeaux II, janvier 1994

Je tiens à remercier $F$. Dubet pour ses conseils et l'attention qu'il a bien voulu porter à ce travail.

(1) Pour une discussion théorique de la notion d'effet établis. sement, $C f$. O. Cousin, "L'effet établissement. Construction d'une problématique ". Revue française de sociologie, juiliet-septembre 1993, 34-3, p. 395-419.

(2) Les facteurs d'organisation retenus dans cette étude ne prétendent pas rendre compte de manière exhaustive de toute la politique des collèges. Nous n'avons pas inclus les indicateurs propres à la gestion de la pédagogique de l'établissement : la composition des classes, les modes de gestion des emplois du temps..., par exemple.

(3) Le collège est le terrain le plus propice à ce type de problématique. Il est l'élément charnière de la scolarité du système éducatif francais, il est aussi le dernier palier offrant la même scolarité à tous les élèves, malgré les différences de prestige et de population entre les établissements. On trouvera en fin d'article une brève présentation des collèges.

(4) La note moyenne de l'élève a été calculée à partir des notes rapportées sur les bulletins scolaires. Nous n'avons retenu que les matières principales (français, mathématiques, histoire-géographie et langue) compte tenu du poids de ces disciplines dans les décisions des conseils de classe. En $4^{\theta}$ et en $3^{\theta}$, les notes de technologie et de la $2^{\theta}$ langue ont été ajoutées. Dans chaque établissement, nous avons effectué un sondage systématique, un élève sur 10, pour calculer la moyenne générale. La différence entre les deux moyennes dépasse rarement un point et elle oscille le plus souvent autour de 0,5 point.
(5) Pour une présentation générale des différents travaux portant sur l'école, Cf. M. Duru-Bellat, A. Henriot-Van Zanten, Sociologie de l'école, Paris, A. Colin, 1992.

(6) $71,5 \%$ des garçons et $80 \%$ des filles passent en $4^{e}$. Cf. M. Duru-Bellat, L'école des filles, Paris, L'harmattan, 1990. C. Baudelot, R. Establet, Allez les filles, Paris, Ed. du Seuil, 1992. G. Felouzis, Le collège au quotidien, Paris, PUF, 1994.

(7) $61 \%$ des enfants d'ouvriers et $90 \%$ des enfants de cadres supérieurs passent en $4^{\theta}$. Cf. M. Duru, A. Mingat, De l'orientation en fin de $5^{\circ}$ au fonctionnement du collège, Tome 1 et 2, Dijon, Cahiers de l'IREDU, $n^{\circ}$ 42, 1985; $n^{\circ} 45,1988$

(8) $89 \%$ des élèves "à l'heure " et $46 \%$ des élèves avec deux ans de retard ou plus passent en $4^{\mathrm{e}}$

(9) $R$ est la racine carrée du coefficient de corrélation $\left(R^{2}\right)$. T est le test de student qui permet d'apprécier le degré de dépendance entre la variable à expliquer et la variable explicative. $T$ est significatif à 0,05 quand il est supérieur à 1,81 .

(10) Ces élèves ont quitté les collèges pour des raisons diverses. Une grande partie a déménagé. Ils sont scolarisés dans un autre département ou dans une autre académie. Une assez faible proportion est restée dans la même aire géographique mais a souhaité changer de collège, quelques-uns sont partis dans le privé. Enfin, un petit nombre a été remercié par l'établissement en fin d'année scolaire ou, quand l'élève a eu 16 ans, il a préféré cesser sa scolarité. Au total ces élèves représentent $8,5 \%$ de la cohorte de départ et $23 \%$ des élèves manquant en $3^{\circ}$

(11) Le système éducatif. Cahiers français. La Documentation française, janvier-février $1991, n^{\circ} 249$, p. 24. 
(12) Repères et références statistiques sur les enseignements et la formation. Ministère de l'éducation nationale et de la culture; D.E.P, 1992. En 1990/1991 le taux de redoublement en fin de 3 était de $9,6 \%$ et le taux de passage en seconde de $64 \%$ pour l'ensemble de la France, soit exactement dans les mémes proportions que pour la cohorte, mais deux années auparavant.

(13) Jusqu'en 1986/1987, le taux de passage en $2^{\text {de }}$ ne dépassait pas $56 \%$ et il augmente pratiquement d'un point tous les ans depuis 1975. Ibid., p 77.

(14) En $5^{e}$ ce sont 368 élèves qui ne se retrouvent pas en $4^{e}$ l'année d'après et, en $3^{e}$, ils sont 379 . La classe de $4^{e}$ représente, quant à elle, $19 \%$ de l'échec. Ces taux sont supérieurs au taux de redoublement et d'orientation vers les filières technologiques, car ils prennent en compte les collégiens qui quittent le collège pour être scolarisés ailleurs.

(15) Ce taux est de $51,1 \%$ pour les élèves arrivés en $6^{\mathrm{e}}$ en 1984 et de $54,8 \%$ (estimation) pour ceux entrés en 1988, soit l'année précédant le début de la recherche. Cf. Le système éducatif, op cit., p. 24.

(16) Si les filles partent elles aussi dans le professionnel, C. Baudelot et R. Establet notent que la mixité existe au sein des établissements mais n'a que très rarement lieu au sein des classes. Allez les filles, op cit.

(17) Le test univarié autorise à rejeter l'hypothèse d'indépendance et le Khi 2 connaît ici sa valeur la plus forte: 102,205, $p<0,0001$.

(18) $5,5 \%$ des enfants de cadres supérieurs partent en LP trois ans après la $5^{\ominus}$ alors que $22 \%$ des enfants d'ouvriers connaissent la méme orientation. Cette différence s'explique à la fois par les meilleures performances des enfants de cadres supérieurs mais aussi, quand les notes sont médiocres, par le fait que l'orientation vers le professionnel est exceptionnellement envisagée. Les parents la refusent et les établissements ne la proposent guère. Cf. M. DuruBellat, A. Mingat, Pour une approche analytique du fonctionnement du système éducatif. Paris, PUF, 1993. J.-M. Berthelot, Ecole, orientation, société, Paris, PUF, 1993.

(19) Les observations de M. Duru et A. Henriot Van-Zanten vont dans le même sens. Les écarts entre les catégories sociales ont diminué depuis l'entrée en vigueur du collège unique. Les enfants d'ouvriers passent a la fois plus souvent en $4^{e}$ et en seconde. Mais, si l'on ne tient compte que des scolarités " normales ", sans redoublement, les inégalités restent inchangées. Sociologie de l'école, op cit

(20) p, test de confiance, est égal à 0,0036

(21) Le statut scolaire distingue les élèves selon leur passé scolaire de $5^{e}$. Lors de la construction de l'échantillon, certains élèves étaient des redoublants de $5^{\theta}(13 \%)$. Cette variable permet d'apprécier les conséquences du redoublement au collège pour le devenir des élèves. Cf. M. Duru-Bellat, J-P. Jarrousse, A. Mingat, "Les scolarités de la maternelle au lycée ", Revue française de sociologie, vol. XXXIV, $n^{\circ} 1$, 1993, p. 43-60.

(22) Seuls les établissements se démarquant significativement de la moyenne sont retenus. Ils sont classés en deux catégories. Les établissements sont "peu sélectifs", quand ils s'écartent positivement de la moyenne dans leur capacité d'amener les élèves de $5^{\theta}$ en seconde en 3 ans, compte tenu de leur recrutement social, J. Renoir, D. Reinhardt et M. Ravel sont dans ce cas. Á l'inverse les établissements sont "sélectifs", quand ils s'écartent négativement de la moyenne. Le Marais, D. Ellington et J. Chardonne sont dans ce cas. Bacchus, du fait de sa particularité, ne sera pas traité ici.

(23) Sur les problèmes de dé-sectorisation et de choix des familles Cf. R. Ballion, Les consommateurs d'école, Paris, Stock, 1982. La bonne école, Paris, Hatier, 1992.
(24) Sur ce point, il est possible de dissocier deux ensembles de recherches. Le premier ensemble accorde une place prépondérante à l'équipe de direction dans la capacité à construire l'identité de l'établissement ou à la mobilisation des acteurs. Cf. D. Paty, Douze collèges en France, Paris, La Documentation française, 1981. R. Ballion, Le lycée, une cité à construire, Paris, Hachette, 1993. F. Dubet, O. Cousin, J-P. Guillemet, * Mobilisation des établissements et performances scolaires ", Revue française de sociologie, 1989, vol. XXX, $n^{\circ} 2$. O. Cousin, J-P. Guillemet, "Variations des performances scolaires et effet d'établissement ". Éducation et formations, $n^{\circ} 31$, avriljuin 1992. Le deuxième ensemble accorde une place plus restreinte à l'équipe de direction et parfois même ne la mentionne pas. $\mathrm{Cf}$. J-L. Derouet, École et justice, Paris, Ed. Métaillé, 1992. M. Duru, A. Mingat, op cit. A. Grisay, Quels indicateurs d'efficacité pour les établissements scolaires, Université de Liège, Service de pédagogie expérimentale, 1989

(25) Ce point est d'autant plus important que rien, dans la formation, ne distingue le chef d'établissement de son adjoint. Le concours de recrutement est identique pour les chefs d'établissement et les adjoints, et le corps de personnel de direction est unifié. En se présentant au concours, les candidats se préparent à assurer la fonction de directeur alors que dans la réalité les lauréats ont peu de chances de le devenir directement. En $1989,14 \%$ des nominés ont démissionné, notamment parce qu'ils ne voulaient pas devenir adjoint. Cf. Le Monde de l'Éducation, $n^{\circ} 175$, octobre 1990.

(26) Cet indicateur a été construit à partir des propos recueillis auprès du personnel éducatif et pédagogique et à partir d'une présence fréquente dans ce lieu essentiel de la vie d'un établissement. Dans certains collèges, le statut des enseignants ou leur appartenance syndicale divise la salle des professeurs, dans d'autres la séparatrion entre les fumeurs et les non-fumeurs recoupent en réalité des conflits larvés entre les professeurs. Les ambiances de salles des professeurs révèlent souvent la nature des liens entre les acteurs et les divisions les plus subtiles cachent en réalité des tensions d'ordre pédagogique et/ou politique. Cf. Y. Dutercq, "Thé ou café ? Ou comment l'analyse de réseau peut aider à comprendre le fonctionnement d'un établissement scolaire", Revue française de Pédagogie $n^{\circ} 95$, avril-mai-juin 91 , p. 81-87.

(27) Dans un grand nombre de cas les projets d'établissement ne sont pas élaborés dans la concertation et le partenariat. Ils ne concernent souvent que le volet éducatif de la mission des collèges. Pour un bilan critique de la politique des projets d'établissement, $C$ f. Le Rapport de l'inspection générale de l'Éducation nationale, Paris, $L a$ Documentation française, 1991. J.-P. Obin, A. Weber, "Les projets d'établissement scolaire: de l'imaginaire à la réalité ", Éducation permanente, $n^{\circ} 87,1987$. R. François, J.P. Obin, Audit des dispositifs concernant les projets d'établissement, Ministère de l'Éducation nationale, mai 1991, ronéotypé. J.-P. Obin. La crise de l'organisation scolaire, Paris, Hachette, 1993.

(28) Projet d'action éducative.

(29) Ils ont été construits dans les années 70 . À J. Renoir, les enfants d'ouvriers représentent $29,5 \%$ des élèves scolarisés dans le collège, les enfants d'employés $20 \%$ et les enfants des professions intermédiaires $22 \%$. $\dot{A}$ D. Reinhardt la répartition est respectivement la suivante : $13,7 \%, 31 \%$ et $20 \%$. Enfin, à $M$. Ravel le collège accueille respectivement $15 \%$ d'enfants d'ouvriers, $30 \%$ d'enfants d'employés et $21,5 \%$ d'enfants de professions intermédiaires. Les données sont sensiblement différentes de celles indiquées en annexes car elles reposent sur l'ensemble des élèves inscrits au collège en 1990 et non pas uniquement sur ceux de $5^{\mathrm{e}}$. 
(30) Cf. Le sondage réalisé par SCP Communication pour Le Monde de l'Éducation auprès 858 personnes. "Les Français jugent leur école ", Le Monde de l'Éducation, $n^{\circ} 208$, octobre 1993. Selon ce sondage, les Français sont plutôt satisfaits de l'école en général ( $93 \%$ d'opinions favorables) et ils souhaitent que leurs enfants poursuivent leurs études au-delà du bac (77\%). Jusqu'à l'école primaire le rôle principal de l'école doit être de développer la person. nalité de l'enfant. À partir du collège, les attentes changent et les Français donnent la priorité à la préparation d'un métier.
(31) Les écarts entre les entants d'ouvriers et les enfants de cadres supérieurs et les catégories intermédiaires pour le passage en seconde sont de 36,3 points en moyenne, à $J$. Renoir ils sont de 29,2 points, à $D$. Reinhardt de 32,1 points et à $M$. Ravel de 17 points.

(32) En moyenne l'écart pour le passage en seconde entre les élèves "à l'heure " et les élèves en retard d'une année est de 41,4 points. II est de 36,2 points à Reinhardt : 51,1 points à Ravel ; 44 points à Renoir ; 37,5 points au Marais ; 34,9 points à Ellington et 39,8 points à Chardonne. 